\title{
Conformally flat spacetimes and Weyl frames
}

\author{
C. Romero, J. B. Fonseca-Neto and M. L. Pucheu \\ ${ }^{a}$ Departamento de Física, Universidade Federal da Paraíba, \\ C.Postal 5008, 58051-970 João Pessoa, Pb, Brazil \\ E-mail: cromero@fisica.ufpb.br
}

October 15, 2018

\begin{abstract}
We discuss the concepts of Weyl and Riemann frames in the context of metric theories of gravity and state the fact that they are completely equivalent as far as geodesic motion is concerned. We apply this result to conformally flat spacetimes and show that a new picture arises when a Riemannian spacetime is taken by means of geometrical gauge transformations into a Minkowskian flat spacetime. We find out that in the Weyl frame gravity is described by a scalar field. We give some examples of how conformally flat spacetime configurations look when viewed from the standpoint of a Weyl frame. We show that in the non-relativistic and weak field regime the Weyl scalar field may be identified with the Newtonian gravitational potential. We suggest an equation for the scalar field by varying the Einstein-Hilbert action restricted to the class of conformallyflat spacetimes. We revisit Einstein and Fokker's interpretation of Nordström scalar gravity theory and draw an analogy between this approach and the Weyl gauge formalism. We briefly take a look at two-dimensional gravity as viewed in the Weyl frame and address the question of quantizing a conformally flat spacetime by going to the Weyl frame.
\end{abstract}

\section{Introduction}

It is well known that the concept of geodesics plays a role of fundamental importance in general relativity as well in any metric theory of gravity. Indeed, an elegant aspect of the geometrization of the gravitational field lies in the geodesics postulate, that is, the statement that light rays and particles moving under the influence of gravity alone follow spacetime geodesics. This fact means that a great deal of information about the motion of particles in a given spacetime is promptly available once one knows its geodesic structure, i.e the set of all geodesics admitted by that spacetime. In general relativity, geodesics are completely determined by the metric properties of the spacetime since it is also assumed that the spacetime geometry has a Riemannian character [1. However, in many other metric theories of gravity one distinguishes between 
metric geodesics and affine geodesics, and so in these theories one must be careful, from the outset, to clearly specify to which kind of geodesics does the geodesic postulates refer [2]. In any case, it is by analyzing the behaviour of timelike and null geodesics, the later determining the light-cone structure, that one is able to predict a series of relativistic phenomena, such as, the existence of the perihelium precession of Mercury's orbit, the deflection of the light by the Sun, the gravitation redshift of light and the gravitational time delay (Shapiro effect). In addition to these, almost all the physics of black holes is obtained by studying the properties of geodesics near the spacetime event horizon. Finally, to explain the existence of a cosmological redshift, the acceleration of the Universe, and many other empirical facts of cosmology all we need is to know the mathematical behaviour of the geodesics corresponding to the underlying cosmological model.

In view of the above, we may conclude that as far as the information conveyed by the geodesic lines of a certain spacetime is concerned one has a certain degree of freedom in the choice of the geometry associated with that space. For instance, in a certain sense it does not seem that the concept of Riemannian curvature is essential for the geometrical description of the gravitational and cosmological phenomena just mentioned. Two distinct geometries sharing the same geodesic structure will give exactly the same description of geodesic-related phenomena, being for this reason indistinguishable from the observational point of view. In this sense the two geometries may be regarded as equivalent. If, in addition, they are related by some kind of mathematical transformation, it may happen that one of them is preferable to the other when we need to do some calculations, or if we want to get a simpler or different picture of physical processes going on. In this paper, we would like to develop further these ideas by considering a kind of interplay between two different frameworks: the geometries of Riemann and Weyl. As we will see, there are circumstances in which it is possible to swift from one to the other while keeping some basic geometric structure invariant. The key notion to understand how such correspondence works is that of geometrical gauge transformation, a concept introduced by $\mathrm{H}$. Weyl in 1918 [3]. The theory developed by Weyl is regarded by many as an elegant generalization of Riemannian geometry, and, in the opinion of some authors, "contains a suggestive formalism and may still have the germs of a future fruitful theory" 4 .

This paper is organized as follows. In Section II, we give a brief introduction to Weyl geometry and introduce the notion of Riemann and Weyl frames. We proceed in Section III to consider how the class of conformally spacetimes is described in the Weyl frame. In Section IV, we show that in the weak field regime the scalar field that appears in the Weyl frame may be identified with the Newtonian gravitational potential. Then, in Section V, we suggest an equation for the Weyl scalar field which may be deduced by varying the Einstein-Hilbert action with respect to the restricted class of conformally flat metrics. Section VI contains a brief discussion of the analogy between the dynamics of the Weyl scalar field and the approach followed by Nordström scalar theory of gravity. In Sections VII we take a brief look at two-dimensional gravity as viewed in the 
Weyl frame. Finally, in Section VII, we address the question of quantizing a conformally flat spacetime by going to the Weyl frame where the problem may be reduced to the quantization of a scalar field in flat spacetime. We summarize our work in Section IX.

\section{Weyl geometry}

Conceived by Weyl in 1918, as an attempt to unify gravity with electromagnetism, in its original form Weyl's theory 3 turned out to be inadequate as a physical theory as was firstly pointed by Einstein soon after the appearance of the theory [5]. As is well known, Einstein's argument was that in a non-integrable Weyl geometry it would not be possible the existence of sharp spectral lines in the presence of an electromagnetic field since atomic clocks would depend on their past history, an effect known as the second clock effect. However, a variant of Weyl geometry, namely, the one in which the Weyl field is integrable, does not suffer from this flaw [6], and, for this reason, has been used in some approaches to gravitation and cosmology in varied contexts [7. Interest in Weyl geometry among physicists has also been increased by the constructive-axiomatic formulation of spacetime theory developed by Ehlers, Pirani and Schild, who demonstrated that if certain axioms, suggested by experience, are satisfied, then one is naturally led to Weyl geometry [8]. On the other hand, there are arguments based on quantum mechanics that seems to rule out non-integral Weyl geometry as a viable [9] framework to describe spacetime, although this point remains controversial [6].

Let us now discuss what kind of geometry Weyl discovered. The essential difference between the Riemann geometry and the Weyl geometry is that in the former one makes the assumption that the covariant derivative $\nabla_{a} g_{b c}$ of the metric tensor $g$ is zero, while in the latter $\nabla_{a} g_{b c}$ is given by

$$
\nabla_{a} g_{b c}=\sigma_{a} g_{b c}
$$

where $\sigma_{a}$ denotes the components of a one-form field $\sigma$ with respect to a local coordinate basis. This represents a generalization of the Riemannian condition of compatibility between the connection $\nabla$ and $g$, which is equivalent to require the length of a vector to remain unaltered by parallel transport 5. If $\sigma$ is an exact form, i.e. $\sigma=d \phi$, where $\phi$ is a scalar field, then we have what is called an integrable Weyl geometry. The triad $(M, g, \sigma)$ where $M$ is a differentiable manifold endowed with a metric $g$ and a Weyl field $\sigma$ will be referred to as a Weyl frame. It is interesting to note that the Weyl condition (11) remains unchanged when we go to another Weyl frame $(M, \bar{g}, \bar{\sigma})$ by performing the following simultaneous transformations in $g$ and $\sigma$ :

$$
\begin{gathered}
\bar{g}=e^{-f} g \\
\bar{\sigma}=\sigma-d f
\end{gathered}
$$

where $f$ is a scalar function defined on $M$. 
Quite analogously to Riemannian geometry, the condition (1) is sufficient to completely determine the Weyl connection $\nabla$ in terms of the metric $g$ and the Weyl one-form field $\sigma$. Indeed, a straightforward calculation shows that one can express the components of the affine connection with respect to an arbitrary vector basis completely in terms of the components of $g$ and $\sigma$ :

$$
\Gamma_{\beta \gamma}^{\alpha}=\left\{\begin{array}{l}
\alpha \\
\beta \gamma
\end{array}\right\}-\frac{1}{2} g^{\alpha \mu}\left[g_{\mu \beta} \sigma_{\gamma}+g_{\mu \gamma} \sigma_{\beta}-g_{\beta \gamma} \sigma_{\mu}\right]
$$

where $\left\{\begin{array}{l}a \\ b c\end{array}\right\}$ represents the Christoffel symbols.

A clear geometrical insight on the properties of Weyl parallel transport is given by the following proposition: Let $M$ be a differentiable manifold with an affine connection $\nabla$, a metric $g$ and a Weyl field of one-forms $\sigma$. If $\nabla$ is compatible with $g$ in the Weyl sense, i.e. if (1) holds, then for any smooth curve $\alpha=\alpha(\lambda)$ and any pair of two parallel vector fields $V$ and $U$ along $\alpha$, we have

$$
\frac{d}{d \lambda} g(V, U)=\sigma\left(\frac{d}{d \lambda}\right) g(V, U)
$$

where $\frac{d}{d \lambda}$ denotes the vector tangent to $\alpha$.

If we integrate the above equation along the curve $\alpha$, starting from a point $P_{0}=\alpha\left(\lambda_{0}\right)$, then we obtain

$$
g(V(\lambda), U(\lambda))=g\left(V\left(\lambda_{0}\right), U\left(\lambda_{0}\right)\right) e^{\int_{\lambda_{0}}^{\lambda} \sigma\left(\frac{d}{d \rho}\right) d \rho}
$$

Putting $U=V$ and denoting by $L(\lambda)$ the length of the vector $V(\lambda)$ at an arbitrary point $P=\alpha(\lambda)$ of the curve, then it is easy to see that in a local coordinate system $\left\{x^{a}\right\}$ the equation (5) reduces to

$$
\frac{d L}{d \lambda}=\frac{\sigma_{\alpha}}{2} \frac{d x^{\alpha}}{d \lambda} L
$$

Consider the set of all closed curves $\alpha:[a, b] \in R \rightarrow M$, i.e, with $\alpha(a)=\alpha(b)$. Then, we have the equation

$$
g(V(b), U(b))=g(V(a), U(a)) e^{\int_{a}^{b} \sigma\left(\frac{d}{d \lambda}\right) d \lambda} .
$$

Now, it is the integral $\int_{a}^{b} \sigma\left(\frac{d}{d \lambda}\right) d \lambda$ that is responsible for the difference between the readings of two identical atomic clocks following different paths. It follows from Stokes' theorem that if $\sigma$ is an exact form, that is, if there exists a scalar function $\phi$, such that $\sigma=d \phi$, then

$$
\oint \sigma\left(\frac{d}{d \lambda}\right) d \lambda=0
$$

for any loop. In other words, in this case the integral $\int_{a}^{b} \sigma\left(\frac{d}{d \rho}\right) d \rho$ does not depend on the path. Since it is this integral that regulates the way atomic clocks run this variant of Weyl geometry does not suffer from the flaw pointed out by Einstein, and we have what is often called in the literature a Weyl integrable manifold. 
Another way to look at (7) is the following. From Frobenius' theorem we know that $\sigma$ is an exact form if and only if $d \sigma=0$. In local coordinates where $\sigma=\sigma_{\alpha} d x^{\alpha}$ this condition reads $F_{\alpha \beta}=\sigma_{\alpha, \beta}-\sigma_{\beta, \alpha}=0$. The quantity $F_{\alpha \beta}=\sigma_{\alpha, \beta}-\sigma_{\beta, \alpha}$, which is non-vanishing in general, is easily shown to be gauge invariant and was interpreted by Weyl as the electromagnetic field in his attempt to geometrize electromagnetism [3]. The 2-form $F=F_{\alpha \beta} d x^{\alpha \wedge} d x^{\beta}$ is called length curvature, so a Weyl integrable manifold is one in which the length curvature $F$ vanishes. Finally, if there is a frame $(M, g, \sigma)$ in which $\sigma=0$, then obviously the geometry of Weyl reduces to Riemann geometry in that frame.

An important feature of Weyl geometry, which will be explored in this paper, is the following mathematical fact. Consider the (affine) geodesic equations

$$
\nabla_{V} V=0
$$

in a certain frame $(M, g, \sigma)$, where $V$ denotes the tangent vector to the geodesic curve. In local coordinates (8) has the form

$$
\frac{d^{2} x^{\mu}}{d \lambda^{2}}+\Gamma_{\alpha \beta}^{\mu} \frac{d x^{\alpha}}{d \lambda} \frac{d x^{\beta}}{d \lambda}=0
$$

where $\Gamma_{\alpha \beta}^{\mu}$ denotes the components of the connection $\nabla, \lambda$ is an affine parameter and $x^{\mu}=x^{\mu}(\lambda)$ represents local parametric equations of the geodesic. Suppose that we change from the frame $(M, g, \sigma)$ to another frame $(M, \bar{g}, \bar{\sigma})$ by performing a gauge transformation in accordance with (2) and (3). It is clear that in each frame the components of $\nabla$, i.e $\Gamma_{\alpha \beta}^{\mu}$ and $\bar{\Gamma}_{\alpha \beta}^{\mu}$, may be expressed in terms of the Christoffel symbols $\left\{\begin{array}{c}\alpha \\ \beta \gamma\end{array}\right\}, \overline{\left\{\begin{array}{c}\alpha \\ \beta \gamma\end{array}\right\}}$ and the Weyl fields $\sigma$ and $\bar{\sigma}$, respectively, as in Eq. (44) . Because $\nabla$ is kept unaltered by the gauge transformations, if $x^{\mu}=x^{\mu}(\lambda)$ is a solution of (8) in the frame $(M, g, \sigma)$, then it is also a solution of that equation in the other frame $(M, \bar{g}, \bar{\phi})$. The geodesic equations are gauge invariant because $\Gamma_{\alpha \beta}^{\mu}=\bar{\Gamma}_{\alpha \beta}^{\mu}$, and the truth of this statement can be easily verified by explicitly using (4).

\section{Conformally flat spacetimes and the Weyl gauge field}

In the light of the concepts just discussed let us consider in this section the class of all conformally flat spacetimes. As we know, a significant number of spacetimes of physical interest predicted by general relativity belong to this class. For instance, it is well known that all Robertson-Walker cosmological models are conformally flat. Explicit conformal transformations taking these to flat spacetime were first given by Infeld and Schild [10]. Let us consider more generally a certain conformally flat spacetime $M$ with a metric $g=e^{\phi} \eta$, where $\eta$ denotes the Minkowski metric and $\phi$ is a scalar function. If the geometry of $M$ is Riemannian we have no Weyl field, and so the components of the affine connection $\Gamma_{\alpha \beta}^{\mu}$ are identical to the Christoffel symbols $\left\{\begin{array}{c}\mu \\ \alpha \beta\end{array}\right\}$. On the other hand, this geometrical configuration is clearly equivalent to the one described in 
terms of Weyl geometry as long as we confine ourselves to the frame $\left(M, e^{\phi} \eta, 0\right)$, to which we will refer as the Riemann frame. Suppose now that we make the gauge transformation (2) and (3) with $f$ replacing $\phi$. In doing so, we arrive at a frame, namely $(M, \eta,-d \phi)$, which will be called the Weyl frame. As we have seen, with respect to geodesics both frames are completely equivalent. Nevertheless, in many aspects the geometry defined by them are entirely distinct. For instance, from the point of view of the Riemannian curvature they are obvioulsly distinct. In effect, in the Riemann frame $\left(M, e^{\phi} \eta, 0\right)$ the manifold $M$ is endowed with a metric that leads to non-zero curvature, while in the Weyl frame $(M, \eta,-d \phi)$ we have a flat spacetime (in the Riemannian sense). Another difference concerns the length of non-null curves or other metric-dependent geometrical quantities since in the two frames we have distinct metric tensors. Null curves, on the other hand, are mapped into null curves. This implies that the light geometry of a conformally flat spacetime is identical to that of Minkowski spacetime, a well known feature of conformal transformations. It is here that the geometrical framework conceived by Weyl comes into play.

As we have already remarked, from the standpoint of geodesics and the lightcone structure we can characterize any cosmological model whose geometry is conformal purely in terms of an integrable Weyl field $\sigma=d \phi$ defined in Minkowski spacetime. It turns out that the passage from the frame $\left(M, e^{\phi} \eta, 0\right)$ to the frame $(M, \eta,-d \phi)$ provides us with a new geometrical picture. For instance, cosmological phenomena, such as the redshift of galaxies or the expansion of the Universe, cease to be necessarily explained by the action of a dynamical curved spacetime . Instead, the dynamics of the Cosmos becomes, in this picture, entirely governed by a gauge field living in a fixed and static spacetime. It is this gauge field, by the way, that controls a new law of parallel displacement and also determines the behaviour of clocks and measuring devices. In other words, to each conformal Riemannian spacetime we can associate a Weyl gauge field in Minkowski spacetime, whose dynamics is ultimately the sole responsible for the motion of particles and light rays. Let us illustrate this point by explicitly calculating the Weyl field from some simple cosmological models.

Consider a Robertson-Walker metric $g$ corresponding to a homogeneous and isotropical cosmological model, written in the form 1

$$
d s^{2}=d t^{2}-A^{2}(t)\left(\frac{d r^{2}}{1-k r^{2}}+r^{2} d \theta^{2}+r^{2} \sin ^{2} \theta d \varphi^{2}\right),
$$

where $k=0, \pm 1$. Taking, for simplicity, the case of flat spatial section $(k=0)$, and defining the so-called conformal time $d \tau=\frac{d t}{A(t)}$, we can rewrite the metric (10) in the conformally flat form

$$
d s^{2}=S^{2}(\tau)\left(d \tau^{2}-d r^{2}-r^{2} d \theta^{2}-r^{2} \sin ^{2} \theta d \varphi^{2}\right),
$$

\footnotetext{
${ }^{1}$ Throughout this paper, except in Section IV, we set $c=1$. We are also adopting the following convention in the definition of the Riemann and Ricci tensors: $R_{\mu \beta \nu}^{\alpha}=\Gamma_{\beta \mu, \nu}^{\alpha}-$ $\Gamma_{\mu \nu, \beta}^{\alpha}+\Gamma_{\rho \nu}^{\alpha} \Gamma_{\beta \mu}^{\rho}-\Gamma_{\rho \beta}^{\alpha} \Gamma_{\nu \mu}^{\rho} ; R_{\mu \nu}=R_{\mu \alpha \nu}^{\alpha}$. In this convention, we will write the Einstein equations as $R_{\mu \nu}-\frac{1}{2} R g_{\mu \nu}-\Lambda g_{\mu \nu}=-\kappa T_{\mu \nu}$, with $\kappa=\frac{8 \pi G}{c 4}$.
} 
where we have defined $S(\tau)=A(t(\tau))$. By carrying out the transformations (22) and (3) with $e^{f}=S^{2}(\tau)$, we can pass from the Riemann frame $(M, g, 0)$ to the Weyl frame $(M, \eta, \sigma)=(M, \eta,-d(2 \ln S(\tau))$. In this frame, the Weyl gauge field is given by the 1-form $\sigma=\frac{-2}{S} \frac{d S}{d \tau} d \tau$, whose components in the coordinate basis are $\sigma_{\mu}=\left(\frac{-2}{S} \frac{d S}{d \tau}, 0,0,0\right)$. In the case of spatially flat Friedmann models, we have $A(t)=A_{0} t^{p}$, where $A_{0}$ is a constant. This functional form of $A(t)$ includes

the so-called matter-dominated $\left(p=\frac{2}{3}\right)$ and the radiation-dominated $\left(p=\frac{1}{2}\right)$ universes, and a possible choice for the conformal time is $\tau=\frac{1}{A_{0}(1-p)} t^{1-p}$ ( $p \neq 1$ ). The expression for the Weyl scalar field $\phi$ as a function of $\tau$ then becomes

$$
\phi(\tau)=-2 \ln A(t(\tau))=-2 \ln \left(a_{0} \tau\right)^{\frac{p}{1-p}},
$$

where we have defined $a_{0}=(1-p)\left(A_{0}\right)^{\frac{1}{p}}$. Thus, for the matter-dominated and the radiation-dominated universes, the Weyl scalar field $\phi(\tau)$ will be given, respectively, by $\phi(\tau)=-4 \ln a_{0} \tau$ and $\phi(\tau)=-2 \ln a_{0} \tau$. We note that in both cases $\phi(\tau)$ has a singularity at $\tau=0$.

Another simple example is given by the de Sitter-Lemaitre cosmological model, whose metric is given by (10), with $k=0$ and $A(t)=A_{0} e^{\sqrt{\frac{1}{3}} t}$, where $\Lambda$ is a positive constant. If we choose the conformal time as $\tau=-\frac{1}{A_{0}} \sqrt{\frac{3}{\Lambda}} e^{-\sqrt{\frac{\Lambda}{3}} t}$, then the scalar field will be given by

$$
\phi(\tau)=-2 \ln \left(-\sqrt{\frac{\Lambda}{3}} \frac{1}{\tau}\right) .
$$

In local coordinates, we have $\sigma_{\mu}=\left(\frac{2}{\tau}, 0,0,0\right)$.

It has been shown recently that the metric of all Robertson-Walker (RW) models $(k=0, \pm 1)$ is conformally flat [13]. For each of these we may apply the above procedure to obtain the Weyl scalar field $\phi$, hence the gauge field $\sigma=d \phi$ , and for $k= \pm 1$ both will be a function of $r$ and $t$.

In view of the above, we see that in the Weyl frame's picture the kinematical behaviour of galaxies in any Robertson-Walker cosmological model is totally determined by the Weyl scalar field $\phi$ while spacetime remains fixed. On the other hand, $\phi$, now looked upon primarily as the conformal factor of a conformally flat spacetime in the Riemann frame, may be considered as a gauging function determining the behaviour of clocks and mesuring rods in a Minkowski spacetime. This second view was noted long ago by Infeld and Schild ([10]). A similar scenario was conceived more recently in which the role of the scalar field is replaced by space and time variation of particle masses. In any of these pictures, the interesting fact is that it is possible to conceive a new scenario, in which the Riemannian curvature ceases to determine the cosmic expansion and other cosmological phenomena, which in our case is the sole responsibility of a scalar field $\phi$. 


\section{Gravity in the Weyl frame}

As we have seen, when we go from one frame $(M, g, \sigma)$ to another frame $(M, \bar{g}, \bar{\sigma})$ through the gauge transformations (2) and (3), the pattern of affine geodesic curves does not change. In particular, the metric geodesics corresponding to a conformally flat spacetime in the Riemann frame $\left(M, e^{\phi} \eta, 0\right)$ are completely indistinguishable from the affine geodesics in the Weyl frame $(M, \eta,-d \phi)$, although in the latter a quite different geometrical picture arises as these curves now lie in a fixed and flat spacetime. This change of perspective might lead, in some cases, to new insights in the description of gravitational phenomena. In the case of a conformally flat spacetime going to the Weyl frame leads to a scenario in which the gravitational field is not associated with a tensor, but with a geometrical scalar field $\phi$ living in a Minkowski background. We can get some insight on the amount of physical information carried by the scalar field $\phi$, in the Weyl frame, by investigating the behaviour of $\phi$, in the regime of weak gravity in the Riemannian frame, where $\phi$ plays the role of a conformal factor. This is the question we want to examine in this section.

Let us recall that a metric theory of gravity is said to possess a Newtonian limit in the non-relativistic weak-field regime if one can derive the Newtonian second law from the geodesic equations as well as the Poisson's equation from the gravitational field equations. Since in Newtonian physics the space geometry is Euclidean, a weak gravitational field in a geometric theory of gravity should manifest itself as a metric phenomenon through a slight perturbation of the Minkowskian spacetime metric. Thus we consider a time-independent metric tensor of the form

$$
g_{\mu \nu} \simeq \eta_{\mu \nu}+\epsilon h_{\mu \nu}
$$

where $n_{\mu \nu}$ is the Minkowski tensor, $\epsilon$ is a small parameter and the term $\epsilon h_{\mu \nu}$ represents a very small time-independent perturbation due to the presence of some matter configuration. For a conformally flat spacetime we have $g_{\mu \nu}=$ $e^{\phi} \eta_{\mu \nu} \simeq(1+\phi) \eta_{\mu \nu}$. If we adopt the Galilean coordinates of special relativity we can write the line element defined by (12) as

$$
d s^{2}=(1+\phi)\left[\left(d x^{0}\right)^{2}-\left(d x^{1}\right)^{2}-\left(d x^{2}\right)^{2}-\left(d x^{3}\right)^{2}\right],
$$

where, as usual, $x^{0}=c t$. Let us now consider the motion of a test particle in the spacetime (13). Since we are working in the non-relativistic regime we will suppose that the velocity $V^{\alpha}=\frac{d x^{\alpha}}{d t}$ of the particle along the geodesic is much less then $c$, so that the $\beta^{\alpha}=\frac{V^{\alpha}}{c}$ will be regarded as very small; so in our calculations only first-order terms in $\epsilon$ and $\beta$ will be kept. Note that in this approximation $\phi$ is regarded as being static and small, i.e. of the same order as $\epsilon$.

Let us now consider the geodesic equations (9)

$$
\frac{d^{2} x^{\mu}}{d s^{2}}+\Gamma_{\alpha \beta}^{\mu} \frac{d x^{\alpha}}{d s} \frac{d x^{\beta}}{d s}=0,
$$

in the Riemann frame $\left(M, e^{\phi} \eta, 0\right)$, with $\Gamma_{\alpha \beta}^{\mu}$ given by (4). Because $\Gamma_{\alpha \beta}^{\mu}$ is invariant with respect to the gauge transformations (2) and (3) the above equations 
look exactly the same in the Weyl frame $(M, \eta,-d \phi)$. On the other hand, in view of the fact that in this frame $\left\{\begin{array}{l}a \\ b c\end{array}\right\}=0$ we have, to first order in $\phi$,

$$
\Gamma_{\mu \nu}^{\alpha}=\frac{1}{2} n^{\alpha \lambda}\left[n_{\lambda \mu} \phi_{, \nu}+n_{\lambda \nu} \phi_{, \mu}-n_{\mu \nu} \phi_{, \lambda}\right] .
$$

Recalling that in this approximation

$$
\left(\frac{d s}{d t}\right)^{2} \cong c^{2}\left(1+\epsilon h_{00}\right)=c^{2}(1+\phi),
$$

it is not difficult to see that, unless $\mu=\nu=0$, the product $\Gamma_{\alpha \beta}^{\mu} \frac{d x^{\alpha}}{d s} \frac{d x^{\beta}}{d s}$ is of order $\beta \phi$ or higher. In this way, the geodesic equations (14) become, to first order in $\phi$ and $\beta$

$$
\frac{d^{2} x^{\mu}}{d s^{2}}+\Gamma_{00}^{\mu}\left(\frac{d x^{0}}{d s}\right)^{2}=0
$$

By taking into account (16) again, the above equation may be written as

$$
\frac{d^{2} x^{\mu}}{d t^{2}}+c^{2} \Gamma_{00}^{\mu}=0
$$

Clearly, for $\mu=0$ the equation (17) reduces to an identity. On the other hand, if $\mu$ is a spatial index a simple calculation gives $\Gamma_{00}^{i}=-\frac{\eta^{i j}}{2} \frac{\partial \phi}{\partial x^{j}}$, and the geodesic equation in this approximation becomes, in three-dimensional vector notation

$$
\frac{d^{2} \vec{X}}{d t^{2}}=-\frac{c^{2}}{2} \vec{\nabla} \phi
$$

which is simply Newton's equation of motion in a classical gravitational field provided we identify the scalar gravitational potential as

$$
U=\frac{c^{2}}{2} \phi
$$

Therefore, as regards to the equation of motion of a test particle, we see that, because Eq. 18 also holds in the Weyl frame, the scalar field $\phi$, when viewed in this frame, plays the role of the Newtonian gravitational potential.

Our next step is to obtain, still in the weak field approximation, a field equation for $\phi$. In order to do that we start with the Einstein equations

$$
R_{\mu \nu}-\frac{1}{2} g_{\mu \nu} R=-\kappa T_{\mu \nu}
$$

and find the expressions for $R_{\mu \nu}$ and $R$ when we take $g_{\mu \nu}=e^{\phi} \eta_{\mu \nu}$. In this way we get 2

$$
\partial_{\mu} \partial_{\nu} \phi-\eta_{\mu \nu} \square \phi-\frac{1}{2} \partial_{\mu} \phi \partial_{\nu} \phi-\frac{1}{4} \eta_{\mu \nu} \partial_{\alpha} \phi \partial^{\alpha} \phi=-\kappa T_{\mu \nu},
$$

\footnotetext{
${ }^{2}$ In $N$ dimensions, for a conformally flat metric $g=e^{\phi} \eta$ the Ricci tensor $R_{\alpha \beta}$ and the scalar curvature $R$ are given by $R_{\alpha \beta}=\frac{1}{2} \eta_{\alpha \beta} \square \phi+\frac{(N-2)}{2}\left(\partial_{\alpha} \partial_{\beta} \phi+\frac{1}{2} \eta_{\alpha \beta} \partial_{\sigma} \phi \partial^{\sigma} \phi-\frac{1}{2} \partial_{\alpha} \phi \partial_{\beta} \phi\right)$ and $R=e^{-\phi}\left[(N-1) \square \phi+\frac{1}{4}(N-1)(N-2) \partial_{\sigma} \phi \partial^{\sigma} \phi\right]$.
} 
where $\square$ denotes the d'Alembertian operator in Minkowski spacetime and $\partial^{\alpha} \phi=$ $\eta^{\alpha \beta} \partial_{\beta} \phi$. At this point let us note that (21) may be regarded as a dynamical equation for a certain scalar field $\phi$ defined in a flat spacetime background. From the standpoint of the Weyl frame observers this could be a perfectly possible interpretation. Note that, although in this frame the Riemannian curvature has been removed away, no information has been lost with regard to geodesic motion.

Let us return to the question of the weak field approximation. Again, recalling that in this approximation $\phi$ is considered static and small, hence neglecting quadratic terms in the derivatives of $\phi$, Eq. (21) reduces to

$$
\partial_{\mu} \partial_{\nu} \phi-\eta_{\mu \nu} \square \phi=-\kappa T_{\mu \nu}
$$

On the other hand, for a perfect fluid configuration (defined in Minkowski spacetime) we have $T_{\mu \nu}=\left(\rho c^{2}+p\right) V_{\mu} V_{\nu}-p \eta_{\mu \nu}$, where $\rho, p$ and $V^{\mu}$ denotes, respectively, the proper rest mass density, pressure and velocity field of the fluid. In a non-relativistic regime we also neglect $p$ with respect to $\rho$, which implies that $T_{00} \simeq \rho c^{2}$. For $\mu=\nu=0$ the above equation gives

$$
\nabla^{2} \phi=-\kappa \rho c^{2}
$$

From (19) and substituting $\kappa=\frac{8 \pi G}{c^{4}}$ we obtain

$$
\nabla^{2} U=-4 \pi G \rho
$$

which is Poisson's equation of Newtonian gravity. This seems to suggest that the scalar field $\phi$ contains, in fact, all information regarding gravity in both Riemannian and Weyl frames.

The Einstein equations written in the form of (21) may be useful to obtain exact solutions for conformally flat spacetimes once we know the energymomentum tensor corresponding to a given matter configuration. Although it is tempting to regard them as dynamical equations for a scalar field $\phi$ in Minkowski spacetime there is a strong objection to such an interpretation: those equations cannot be derived from an action principle. Clearly, a scalar field must obey a scalar equation, while (21) are tensor equations. There is, however, a scalar equation naturally associated with $\phi$, which can be obtained by contracting (21) with the Minkowski metric $\eta_{\mu \nu}$. This leads to

$$
\square \phi+\frac{1}{2} \phi^{, \mu} \phi_{, \mu}=\frac{\kappa}{3} T,
$$

where $T=\eta^{\mu \nu} T_{\mu \nu}$ denotes the trace of $T_{\mu \nu}$ with respect to Minkowski metric $\eta_{\mu \nu}$. Of course, the above equation is equivalent to the scalar equation $R=\kappa T$, obtained by taking the trace of (20) with respect to $g_{\mu \nu}=e^{\phi} \eta_{\mu \nu}$. It is interesting that this equation can also be derived from an action principle, as we will show next. 


\section{A scalar equation for the Weyl scalar field}

Let us leave general relativity for a while and speculate how one would formulate a strict scalar field theory of gravity in which the Weyl scalar field, a purely geometrical entity that defines the affine connection, would play the role of the gravitational field in a Minkowski background. We have seen in the previous section that, as long as we restrict ourselves to conformally flat spacetimes, gravity may effectively be described by a scalar field in Minkowski spacetime. An interesting approach to this question would be to start with the formulation of general relativity in terms of a variational principle. Thus, let us consider the Einstein-Hilbert action of the gravitational field in the presence of matter

$$
S=\int_{\Omega} \sqrt{-g}\left(R+\kappa L_{m}\right) d^{4} x
$$

where $R$ is the scalar curvature, $L_{m}$ denotes the Lagrangian density of matter, $\kappa$ is the Einstein constant and $\Omega$ is a regular domain in $M$. The duality between the Riemann frame $\left(M, e^{\phi} \eta, 0\right)$ and the Weyl frame $(M, \eta,-d \phi)$ seems to suggest that in the variation of the functional (23) we should consider only variations $\delta g_{\mu \nu}$ restricted to the class of conformally flat spacetimes, that is, variations of the form

$$
\delta g_{\mu \nu}=\delta\left(e^{\phi} \eta_{\mu \nu}\right)=e^{\phi} \eta_{\mu \nu} \delta \phi .
$$

It is then not difficult to verify that the variation $\delta S$ in the action induced by (24) will be given by

$$
\delta S=-\int_{\Omega} e^{\phi}\left(R_{\mu \nu}-\frac{1}{2} g_{\mu \nu} R+\kappa T_{\mu \nu}\right) \eta^{\mu \nu} \delta \phi d^{4} x,
$$

where we have taken into account that $g^{\mu \nu}=e^{-\phi} \eta^{\mu \nu}, \delta g^{\mu \nu}=-e^{-\phi} \eta^{\mu \nu} \delta \phi$, $\sqrt{-g}=e^{2 \phi}$ and, as usual, the energy-momentum tensor $T_{\mu \nu}$ is defined by $\delta \int_{\Omega} \sqrt{-g} L_{m} d^{4} x=\int_{\Omega} \sqrt{-g} T_{\mu \nu} \delta g^{\mu \nu} d^{4} x$. Since $\delta \phi$ is arbitrary, the condition $\delta S=0$ implies

$$
R=\kappa \mathcal{T},
$$

where $\mathcal{T}=g^{\mu \nu} T_{\mu \nu}=e^{-\phi} \eta^{\mu \nu} T_{\mu \nu}$ denotes the trace of the energy-momentum tensor with respect to the metric $g_{\mu \nu}$. We see, then, that we can derive (26) by just taking the variation of (23) with respect to $\phi$ in the restricted class of conformally spacetime metrics. On the other hand, if we express the curvature scalar $R$ in terms of $\phi$ we get

$$
R=3 e^{-\phi}\left(\square \phi+\frac{1}{2} \phi^{, \mu} \phi_{, \mu}\right),
$$

where we are now using the notation $\phi_{, \mu}=\partial_{\mu} \phi$ and $\phi^{, \mu}=\eta^{\mu \nu} \phi_{, \nu}$. Substituting (27) into (26) yields again

$$
\square \phi+\frac{1}{2} \phi^{, \mu} \phi_{, \mu}=\frac{\kappa}{3} T
$$


where, as before, $T=\eta^{\mu \nu} T_{\mu \nu}$ denotes the trace of $T_{\mu \nu}$ with respect to Minkowski metric $\eta_{\mu \nu}$. We now can look at this equation from the point of view of the Weyl frame $(M, \eta,-d \phi)$ and regard it as a dynamical field equation for the scalar field $\phi$. Finally, we note that if we define the new scalar field variable $\psi=e^{\frac{\phi}{2}}$ we can get rid of the quadratic term $\frac{1}{2} \phi^{, \mu} \phi_{, \mu}$, and hence Eq.(22) may be put in the simpler form

$$
\frac{\square \psi}{\psi}=\frac{\kappa}{6} T .
$$

At this point, we think it is worth mentioning that the scalar equation (22) may be derived from varying the action

$$
S=\int d^{4} x e^{\phi}\left[\partial_{\mu} \phi \partial^{\mu} \phi+\frac{2}{3} \kappa T\right]
$$

with respect to the scalar field $\phi$. It is also interesting to note that scalar gravity does not couple with a purely radiating electromagnetic field since in this case $T=0$, which is consistent with the fact that in the Riemann frame spacetime is conformally flat, and so null geodesics representing light rays consist of straight lines.

We would like to conclude this section with two comments. First, let us note that Eq. (22) is a direct consequence of the Einstein's field equations

$$
R_{\mu \nu}-\frac{1}{2} g_{\mu \nu} R=-\kappa T_{\mu \nu}
$$

which for conformally flat spacetimes in the Riemann frame takes the form of Eq. (21).Therefore, any solution of the Einstein equations is also a solution of (22) since this equation comes from (26). Nevertheless, the converse is not true, and this implies that the class of solutions of (22) is larger than the class of solutions of (21). However, because (22) is much easier to solve, it may sometimes be helpful in getting solutions of (21).

Finally, let us note that if we include the cosmological constant $\Lambda$ in the Einstein equations (31), then (21) and (22) will become, respectively,

$$
\begin{gathered}
\partial_{\mu} \partial_{\nu} \phi-\eta_{\mu \nu} \square \phi-\frac{1}{2} \partial_{\mu} \phi \partial_{\nu} \phi-\frac{1}{4} \eta_{\mu \nu} \partial_{\alpha} \phi \partial^{\alpha} \phi-\Lambda \eta_{\mu \nu} e^{\phi}=-\kappa T_{\mu \nu}, \\
\square \phi+\frac{1}{2} \phi^{, \mu} \phi_{, \mu}+\frac{4}{3} \Lambda e^{\phi}=\frac{\kappa}{3} T .
\end{gathered}
$$

It is not difficult to see that in terms of $\psi=e^{\frac{\phi}{2}}$ (32) reads

$$
\square \psi+\frac{2}{3} \Lambda \psi^{3}=\frac{\kappa}{6} \psi T
$$

Incidentally, we note that in vacuum $(T=0)$ the above equation is a non-linear Klein-Gordon equation, which has exact solutions in the form of a travelling wave 14. 


\section{Weyl frames and scalar theories of gravity}

As is well known, scalar theories of gravity first appeared with the work G. Nordström [15, in his attempts to formulate a special relativistic theory of gravitation [16]. In the second of these attempts, he postulates the following equation for the gravitational field:

$$
\square \Phi=-4 \pi G \Phi \eta^{\alpha \beta} T_{\alpha \beta},
$$

where $T_{\alpha \beta}$ is the energy-momentum tensor of the matter content and spacetime is flat [17. Norsdström also assumed that the motion of test particles would obey the equation

$$
\dot{V}_{\alpha}=-\partial_{\alpha} \Phi-\dot{\Phi} V_{\alpha},
$$

where dot denotes derivative with respect to proper time in Minkwoski space. This theory, as was shown by Einstein and Fokker [18, may be formulated in terms of a metric theory of gravity whose field equation is

$$
R=-24 \pi G \mathcal{T},
$$

with $\mathcal{T}$ as defined in the previous section. There is a supplementary condition, namely that the Weyl tensor constructed from $g_{\alpha \beta}$ vanishes or, equivalently, that spacetime is conformal. In the Einstein-Fokker approach, the equation of motion (34) is replaced by a geodesic equation with respect to the metric $g_{\alpha \beta}=\Phi^{2} \eta_{\alpha \beta}$, which also defines proper time in this curved space. It is interesting to note that both approaches may be formally considered as leading to the same theory formulated in different frames: Einstein's in the Riemann frame $\left(M, \Phi^{2} \eta, 0\right)$, and Nordström's in the Weyl frame $\left(M, \eta,-\frac{2}{\Phi} d \Phi\right)$. Note that by putting $\Phi=e^{\frac{\phi}{2}}=\psi$ we see that Eq. (33) is equivalent to (22) or to (29).

\section{Weyl frames and two-dimensional gravity}

It is a well known result of differential geomety that in two dimensions all spaces are conformally flat. This fact makes the duality between the Riemann and Weyl frames in two dimensions completely general. In other words, geometrical phenomena taking place in a curved two-dimensional space may be described in a flat space endowed with a Weyl connection by simply changing frames. This feature of two-dimensional geometry may perhaps be explored in the context of two-dimensional gravity models. In this Section, we would like to glimpse gravity in this dimensionality in a Weyl frame.

Lower-dimensional theories of gravity, mainly in connection with the quantization of the gravitational field program, have atracted the attention of many physicist during the last forty years [20]. One of the most popular versions of two-dimensional gravity, which reduces to Newtonian gravity in two-dimensional in non-relativistic and weak field regime, postulates the field equation [21]

$$
R+\Lambda=8 \pi G \mathcal{T}
$$


Because in two dimensions the Riemann tensor is completely determined by the curvature scalar $R$, the above equation seems to be the natural analogue of the Einstein equations with the cosmological constant $\Lambda$. Let us note that in this theory the conservation laws $T_{; \nu}^{\mu \nu}=0$ cannot be deduced from the field equation (35) and has to be separately postulated.

For a conformally flat metric $g=e^{\phi} \eta$ the curvature scalar $R$ is given, in two dimensions, by $R=e^{-\phi} \square \phi$. We thus can write Eq. (35) as

$$
\square \phi+e^{\phi} \Lambda=8 \pi G T
$$

where, as in (22) and (32), $T=\eta^{\mu \nu} T_{\mu \nu}$ denotes the trace of $T_{\mu \nu}$ with respect to Minkowski metric $\eta_{\mu \nu}$. As before, in the Weyl frame Eq.(36) may be interpreted as a dynamical equation for the Weyl scalar field $\phi$, which again plays the role of the gravitational field. Incidentally, in the absence of the cosmological constant Eq.(36) becomes

$$
\square \phi=8 \pi G T
$$

which is a wave equation with source term.

\section{Weyl frames and quantum gravity}

As a mathematical tool conformal transformations have been widely used in general relativity, particularly in the theory of asymptotic flatness [22] They also have been employed in connection with scalar-tensor theories of gravity. In fact there has been a long debate on whether different frames related by conformal transformations have equivalent physical meaning [23]. In different conformal frames, the description of physical phenomena may look different, though they are related to each other by a mathematical transformation. To our knowledge this debate has, apparently, being restricted to the context of classical physics. In this section we would like to very briefly discuss some ideas on the subject of quantization of the gravitational field in connection with the notion of Weyl frames. Surely we are not considering the problem of quantum gravity in its generality as we are restricted to the class of conformally flat spacetimes.

Quantum gravity is widely recognized as one of the most difficult and challenging problems of theoretical physics. There is currently a vast body of knowledge which includes several approaches to this area of research, but as far as we know none of them has been entirely succesful to date. Among the most popular of these are string theory [24] and loop quantum gravity [25]. There is, however, a feeling among theorists, that a final theory of quantum gravity, if indeed there is one, is likely to emerge gradually and will ultimately be a combination of different theoretical frameworks. In this spirit let us indulge ourselves for a while in raising some questions concerning the possibility of using of the concept Weyl frame as a way of looking at the problem of quantization of the gravitational field in some particular cases.

We begin by considering the class of all conformally flat spacetimes, i.e. those for which $C_{\alpha \beta \mu \nu}=0$, where $C_{\alpha \beta \mu \nu}$ denote the components of Weyl's 
conformal tensor. This condition reduces the number of independent metric components to only one, and also implies that $g=e^{\phi} \eta$, where $\phi$ is a scalar field. Now, this kind of geometry, whose number of degrees of freedom has been drastically reduced and fixed a priori is an example of what has been called a prior geometry [17. For such spacetimes all information about the gravitational field is encoded in the scalar field $\phi$, so it seems not unreasonable to expect that any quantum aspect emerging in the process of quantization of the gravitation field should somehow involve this field, even though it may be objected that gravitons generated by $\phi$ would be spin- 0 particles . Moreover, one would also expect that the correspondence between the Riemann and Weyl frames, which holds for conformally flat spacetimes at the classical level, would be preserved at the quantum level. If this is true, then it would make sense to carry over the scheme of quantization from the Riemann frame to the Weyl frame. Nevertheless, although in the Weyl frame the spacetime geometry has no longer any degrees of freedom, the scalar field $\phi$ is still a repository of physical information. It would then seem plausible to treat $\phi$ as a physical field. We then are left with a situation which is typical of the ones considered by quantum field theory in flat spacetime. In fact this is not so unusual as in perturbative string theory spacetime is also treated as an essentially classical background 24], not to mention that Feymann used to hold the view that a quantum theory of gravitation should lead to massless spin-2 quanta coupled to matter in flat Minkowski spacetime [26]. Let us suppose that we succumb to the temptation of pursueing this analogy more seriously and proceed to the quantization of the Weyl scalar field in Minkowski spacetime. Many questions would arise at this point. For instance, to quantize the scalar field $\phi$ we need to know what the dynamics of $\phi$. We have seen that the dynamical equations of $\phi$ are given by (21) of which (22) is a consequence. What would happen, however, if we provisionally regarded (22), which may be derived from the action (30), as the fundamental equation to be quantized? We leave this and other questions for future work.

\section{Conclusion}

In this paper we have developed the idea that there is a connection between two different geometrical descriptions of conformally flat spacetimes. With the help of concepts borrowed from Weyl geometry we have shown that some geometrical phenomena taking place in a Riemannian curved spacetime may be described in Minkowski flat space, in such a way that the curvature of the first is replaced, in the second, by a dynamical scalar field $\phi$. This field has a geometrical character as it gives rise to a non-Riemannian affine connection in the flat space. We are thus left with two different pictures. Accordingly, we can reinterpret the essential facts of Robertson-Walker cosmology in terms of a flat spacetime cosmology, in which the motion of galaxies takes place in Minkowski spacetime and is determined by a scalar field. A similar scenario was conceived some years ago, but in a different context in which there is no scalar field but 
the particle masses may depend on space and time [11. We have also discussed other concrete situations in which the two mathematically equivalent descrip-

tions seem to lead to different physical pictures, and these are scalar gravity and two-dimensional gravity. Finally, we slightly touch on the possibility of investigating whether one could apply the mathematical formalism connecting conformally flat spacetimes and scalar fields to the quantization of gravity in Minkowski spacetime.

\section{Acknowledgement}

The authors would like to thank CNPq and CLAF for financial support.

\section{References}

[1] Let us recall that in general relativity the geodesic postulate is not an independent postulate as it follows logically from the Einstein field equations. See, for instance, A. Einstein, L. Infeld and B. Hoffmann, Ann. Math. 39 (2), 65 (1938).

[2] For a nice review of alternative theories of gravity see C. M. Will, Theory and Experiment in Gravitation Physics (Cambridge University Press, 1993).

[3] H. Weyl, Sitzungsber. Preuss. Akad. Wiss., 465 (1918). H. Weyl, Space, Time, Matter (Dover, New York, 1952).

[4] R. Adler, M. Bazin and M. Schiffer, Introduction to General Relativity, Ch. 15, (McGraw-Hill, 1975)

[5] See, for instance, W. Pauli, Theory of Relativity (Dover, New York, 1981). See, also, L. O'Raiefeartaigh and N. Straumann, Rev. Mod. Phys. 72, 1 (2000).

[6] V. Perlick, Class. Quantum Grav. 8, 1369 (1991).

[7] M. Novello and H. Heintzmann, Phys. Lett. A 98, 10 (1983). M. Novello, L.A.R. Oliveira, J.M. Salim, E. Elbas, Int. J. Mod. Phys. D1 (1993) 641677. J. M. Salim and S. L. Sautú, Class. Quant. Grav. 13, 353 (1996). H. P. de Oliveira, J. M. Salim and S. L. Sautú, Class.Quant.Grav. 14, 2833 (1997). V. Melnikov, Classical Solutions in Multidimensional Cosmology in Proceedings of the VIII Brazilian School of Cosmology and Gravitation II (1995), edited by M. Novello (Editions Frontières) pp. 542-560, ISBN 2-86332-192-7. K.A. Bronnikov, M.Yu. Konstantinov, V.N. Melnikov, Grav.Cosmol. 1 (1995). O. Arias, R. Cardenas and I.Quiros, Nucl. Phys. B643, 187 (2002). J. Miritzis, Class. Quantum .Grav. 21, 3043 (2004). J. Miritzis, J.Phys. Conf. Ser . 8,131 (2005). M. Israelit, Found. Phys. 35, 1725 (2005). F. Dahia, G. A. T. Gomez and C. Romero, J. Math.Phys. 
49, 102501 (2008). J. E. Madriz Aguilar and C. Romero, Found. Phys. 39, 1205 (2009).

[8] J. Ehlers, F. Pirani and A. Schild, General Relativity, ed. L. O. Raifeartaigh (Oxford University Press, London, 1972).

[9] J. Audretsch, Phys. Rev. D 27, 2872 (1983). J. Audretsch, F. Gäller and S. Norbert, Commun. Math. Phys (1984).

[10] L. Infeld and A. Schild, Phys. Rev. 68, 250 (1945).

[11] J. Narlikar and H. Arp, Astrophys. J. 405, 51 (1992).

[12] See, for instance R. d'Inverno, Introducing Einstein's Relativity, Ch. 22, p.318 ( Clarendon Press, 1992).

[13] M. Ibison, J. Math. Phys 48, 122501 (2007).

[14] A. D. Polyanin and V. F. Zaitsev, Handbook of Nonlinear Partial Differential Equations (Chapmann \& Hall, 2004). I. Matsuno, J. Math. Phys. 28, 2317 (1987)

[15] G. Nordström, Phys. Zeit. 13, 1126 (1912). G. Nordström, Ann. der Phys. 40, 856 (1913). G. Nordström, Ann. der Phys. 42, 533 (1913).

[16] For a review of special-relativisic theories of gravitation, see J. G. Whithrow and G. E. Murdoch, Relativistic theories of gravitation in Vistas in Astronomy 6, 1, Ed. A. Beer (Oxford, 1965).

[17] Here we consider a slightly different version of Nordström's theory given in C. W. Misner, K. S. Thorne and J. A. Wheeler, Gravitation, Ch. 17 (Freeman, San Francisco, 1073).

[18] A. Einstein and A. D. Fokker, Ann. der Phys. 44, 321 (1914).

[19] S. L. Shapiro and S. A. Teukolsky, Phys. Rev. D47, 1529 (1993). H. Dehnen and H. Frommert, Int. J. of Theor. Phys. 29, 361 (1990). K. Watt and C. Misner, arXiv:gr-qc/9910032 F. Girelli, S. Liberati and L. Sindoni, Phys. Rev. D79, 044019 (2009).

[20] For a review, see, for instance, J. D. Brown, Lower Dimensional Gravity (World Scientific, 1988) and references therein.

[21] A. E. Sikkema and R. B. Mann, Class. Quantum Grav. 8, 219 (1991).

[22] R. M. Wald, General Relativity (Chigago University Press, Chigago, 1984).

[23] V. Faraoni, E. Gunzig and P. Nardone, Fund. Cosm. Phys. 20, 121 (1999). V. Faraoni and S. Nadeau, Phys. Rev. D75, 023501 (2007). V. Faraoni, Cosmology in Scalar-Tensor Gravity, Ch.2 (Kluwer Academic Publishers, 2004). Y. Fujii and K. Maeda, The Scalar-Tensor of Gravitation, Ch.1 (Cambridge University Press, 2003). 
[24] M. B. Green, J. Schwarz and E. Witten, Superstring theory, Volume 1 (Cambridge University Press, 1988)

[25] See, for instance, C. Rovelli, Quantum Gravity (Cambridge University Press, 2007). C. Kiefer, Quantum Gravity (Oxford University Press, 2nd edition, 2007).

[26] R. P. Feynmann, F. B. Morinigo and W. G. Wagner, Feynmann Lectures on Gravitation (Addison-Wesley, 1995). 